\title{
CODAZZI TENSORS AND REDUCIBLE SUBMANIFOLDS
}

BY

IRL BIVENS

\begin{abstract}
An integral formula is derived for Codazzi tensors of type $(k, k)$. Many of the classical Minkowski type integral formulas then become special cases of this one. If $M$ is a submanifold of Euclidean space and $\pi$ is a parallel distribution on $M$ then each leaf of $\pi$ is a submanifold of Euclidean space with mean curvature normal vector field $\eta$. Using the above integral formula we show that the integral of $|\eta|^{2}$ over $M$ is bounded below by an intrinsic constant and we give necessary and sufficient conditions for equality to hold. The reducible surfaces for which equality holds are characterized and related results concerned with Riemannian product manifolds are proved.

Parallel tensors of type $(1,1)$ are characterized in terms of the de Rham decomposition. It is shown that if $M$ is irreducible and $A$ is a parallel tensor of type $(1,1)$ on $M$ which is not multiplication by a constant then $M$ is a Kaehler manifold. Some further results are derived for manifolds whose simply connected cover is Kaehler.
\end{abstract}

Acknowledgements. Most of the results of this paper were contained in my thesis written under the direction of Professor Patrick Eberlein at the University of North Carolina at Chapel Hill. It is a pleasure to acknowledge here my debt to Professor Eberlein, both for his guidance in matters mathematical, and for his unfailing support and encouragement.

1. Introduction. The purpose of this paper is to extend and give proofs of the results announced in [2] concerning Codazzi tensors and reducible submanifolds of Euclidean space.

Let $(M,\langle\rangle)$ denote a Riemannian manifold with $S$ a tensor of type $(k, k)$ on $M$. We say $S \in \Gamma\left[\right.$ End $\left.\Lambda^{k}(T M)\right]$ provided $S$ is alternating in the first $k$ and in the last $k$ indices. As the notation suggests, at each point $x \in M, S_{x}$ may be identified with an element of End $\Lambda^{k} T_{x} M$. Given $S$ in $\Gamma\left[\right.$ End $\Lambda^{k}(T M)$ ] and $T$ in $\Gamma\left[\right.$ End $\left.\Lambda^{j}(T M)\right]$ we may define $S * T \in \Gamma\left[\right.$ End $\left.\Lambda^{k+j}(T M)\right]$ by wedging the respective covariant and contravariant components of $S$ and $T$. The multiplication, *, is associative and commutative. Let $\nabla$ denote the Riemannian connection on the full tensor algebra of $M$.

Definition 1.1. A Codazzi tensor of type $(k, k)$ will be an element $S$ of $\Gamma\left[\right.$ End $\left.\Lambda^{k}(T M)\right]$ such that

$$
0=\Sigma(-1)^{j+1}\left(\nabla_{X_{j}} S\right)\left(X_{1} \wedge X_{2} \wedge \cdots \wedge X_{j-1} \wedge X_{j+1} \wedge \cdots \wedge X_{k+1}\right)
$$

for all $C^{\infty}$ vector fields $X_{1}, X_{2}, \ldots, X_{k+1}$ on $M$.

Received by the editors October 20, 1980.

1980 Mathematics Subject Classification. Primary 53A07, 53C55, 58A30; Secondary 53A45, 58C40. 
A routine computation shows that if $S$ and $T$ are Codazzi tensors of types $(k, k)$ and $(j, j)$ respectively then $S * T$ is a Codazzi tensor of type $(k+j, k+j)$.

EXAMPLES. (a) Let $M$ be an oriented hypersurface of Euclidean space with $N$ a unit normal vector field on $M$. The 'shape operator' is a tensor $T$ of type $(1,1)$ on $M$ defined by $T(X)=-\tilde{\nabla}_{X} N$, where $X$ is a tangent vector to $M$ and $\tilde{\nabla}$ denotes the Euclidean covariant derivative. The Codazzi equations for $M$ imply $T$ is a Codazzi tensor of type $(1,1)$. More generally if $M$ is a submanifold of Euclidean space and $N$ is a unit normal vector field on $M$ which is parallel in the normal bundle then $T$ (defined by the same equation) is a Codazzi tensor of type $(1,1)$. In either case $S=T^{k}=T * T * \cdots * T$ ( $k$ times) is a Codazzi tensor of type $(k, k)$.

(b) The curvature operators $R_{2 k} \in \Gamma\left[\right.$ End $\left.\Lambda^{2 k}(T M)\right]$ are defined by $\left\langle R_{2}(X \wedge Y)\right.$, $Z \wedge W\rangle=\langle R(X, Y) Z, W\rangle$ and $R_{2 k}=R_{2} * R_{2} * \cdots * R_{2}$ ( $k$ times) where $R$ denotes the curvature tensor of $M$. The second Bianchi identity implies $R_{2}$ is a Codazzi tensor of type $(2,2)$ and thus $R_{2 k}$ is a Codazzi tensor of type $(2 k, 2 k)$.

Suppose $M$ is an $n$ dimensional compact, oriented, Riemannian manifold without boundary and $g: M \rightarrow \mathbf{R}^{N}$ is an isometric immersion. If $S$ is a Codazzi tensor of type $(k, k)$ then

THEOREM 2.1. $\int_{M}(n-k)$ trace $S+$ trace $S * A d V=0$ where $d V$ denotes the volume element of $M$ and $A$ is a symmetric tensor of type $(1,1)$ depending on the immersion $g$.

By applying Theorem 2.1 to the aforementioned examples of Codazzi tensors we recover the integral formulas of [3], [8], and [13].

If $\pi$ is a $C^{\infty}$ distribution on $M$ which is invariant under parallel translation along any path then $\pi$ is said to be parallel. In terms of the Riemannian connection, a distribution $\pi$ is parallel iff $\nabla_{X} Y$ is tangent to $\pi$ for any $C^{\infty}$ vector fields $X$ and $Y$ on $M$ with $Y$ tangent to $\pi$. It immediately follows that if $\pi$ is parallel then $\pi$ is integrable with totally geodesic leaves and that the distribution orthogonal to $\pi$ is also parallel. If $M$ possesses a parallel distribution $\pi$ with $0<\operatorname{dim} \pi<\operatorname{dim} M$ then $M$ is said to be reducible. If no such distribution exists then $M$ is said to be irreducible. A complete, simply connected Riemannian manifold $M$ is isometric to a Riemannian product $M_{0} \times M_{1} \times \cdots \times M_{k}$ (the de Rham decomposition) where $M_{0}$ is a Euclidean space and $M_{1} \cdots M_{k}$ are complete simply connected irreducible Riemannian manifolds. Such a decomposition is unique up to the order of the factors [11].

Let $M$ and $g: M \rightarrow \mathbf{R}^{N}$ satisfy the hypotheses of Theorem 2.1 with $\pi$ a parallel distribution on $M$. Each leaf of $\pi$ is isometrically immersed into Euclidean space by $g$ and given $p \in M$ we denote by $\eta(p)$ the mean curvature normal vector at $p$ for the corresponding immersed leaf of $\pi$. Using Theorem 2.1 and an argument originally carried out by $\mathrm{R}$. Reilly [13] for the case $\pi=T M$ we prove

THEOREM 3.5. $\int_{M}|\eta|^{2} d V \geqslant\left(\lambda_{1}\right.$ vol $\left.M\right) / n$ where $\lambda_{1}$ denotes the first eigenvalue of the Laplacian on $M$. Equality holds iff both $M$ and the leaves of $\pi$ are minimally immersed into a sphere of radius $R=\left(n / \lambda_{1}\right)^{1 / 2}$. 
Denote by the triple $(M, g, \pi)$ a manifold $M$ and an isometric immersion $g: M \rightarrow \mathbf{R}^{N}$ as above, with $\pi$ a parallel distribution on $M$. Let $S$ denote the set of such triples for which equality holds in Theorem 3.5. In $\$ 3$ we prove a number of results concerned with product manifolds and the class $S$. For example

Corollary 3.11. If $\left(M_{1} \times M_{2}, g, \pi\right) \in S$ then

(a) $g$ splits as a product immersion $g=g_{1} \times g_{2}$;

(b) $\left(M_{i}, g_{i}, T M_{i}\right) \in S, i=1,2$;

(c) $\lambda_{1}\left(M_{1}\right)=\lambda_{1}\left(M_{2}\right)$.

The triples $(M, g, \pi) \in S$ with $\operatorname{dim} M=2$ and $\operatorname{dim} \pi=1$ are characterized by

TheOrem 3.13. If $(M, g, \pi) \in S$ with $\operatorname{dim} M=2$ and $\operatorname{dim} \pi=1$ then $M$ is isometric to the flat torus $\mathbf{R}^{2} / \alpha\left(\mathbf{Z}^{2}\right), \alpha=2 \pi /\left(\lambda_{1}\right)^{1 / 2}$, and $g$ splits as a product embedding of $M$ into a 4 dimensional flat of $\mathbf{R}^{N}$. Furthermore $\pi$ is generated by the lines of slope 1 or -1 in $\mathbf{R}^{2}$.

A particularly interesting class of Codazzi tensors is given by the space of parallel tensors of type $(1,1)$ on a Riemannian manifold. In Proposition 4.5 parallel tensors of type $(1,1)$ on a complete simply connected Riemannian manifold are shown to be products of parallel tensors of type $(1,1)$ on each of the irreducible factors of the de Rham decomposition of the manifold. On an irreducible Riemannian manifold $M$, there is a close connection between this space of parallel tensors and the existence of a Kaehler structure for $M$.

Proposition 4.1. If $M$ is irreducible and $A$ is a parallel tensor of type $(1,1)$ on $M$ then either $A=\alpha I$ or $A=\alpha I+\beta J, \alpha, \beta \in \mathbf{R}, J$ a Kaehler structure (depending on A) on $M$.

In fact, on an irreducible Riemannian manifold the space, $Z$, of parallel tensors of type $(1,1)$ is a finite dimensional real division algebra and thus

Proposition 4.2. If $M$ is irreducible then $Z$ is isomorphic to one of the following: (i) the real numbers $\mathbf{R}$, (ii) the complex numbers $\mathbf{C}$, (iii) the quaternions $\mathbf{H}$.

If $M$ is irreducible and either $\operatorname{dim} M$ is not divisible by 4 or $M$ is not Ricci flat then $Z$ is at most 2 dimensional. In particular if $M$ is Kaehler then it has a unique (up to sign) Kaehler structure. If $M$ is not assumed Kaehler but can be covered by a Kaehler manifold then interesting consequences still follow.

Proposition 4.3. Let $M$ be an $n$ dimensional irreducible Riemannian manifold which is covered by a Kaehler manifold.

(a) If $M$ is compact with finite fundamental group and if either $n$ is not divisible by 4 or $M$ is not Ricci flat then the $4 k$ th Betti numbers of $M$ are nonzero.

(b) If $n$ is not divisible by 4 and $M$ is oriented then $M$ is itself Kaehler.

(c) If $n$ is divisible by 4 and $M$ is not Ricci flat then $M$ is oriented and is either Kaehler or is doubly covered by a Kaehler manifold. 
As a consequence of Proposition 4.3 and a theorem of Kobayashi we have

COROLlARY 4.4. Let $M$ be a compact irreducible Riemannian manifold which is covered by a Kaehler manifold. If the Ricci tensor of $M$ is positive definite then either $M$ is simply connected or $\pi_{1}(M)=\mathbf{Z} /(2)$.

2. An integral formula for Codazzi tensors. Let $M$ be a compact oriented $n$ dimensional Riemannian manifold without boundary with $g: M \rightarrow \mathbf{R}^{N}$ an isometric immersion. Define a symmetric tensor $A$ of type $(1,1)$ on $M$ via the formula

$$
\langle A(X), Z\rangle=\langle\mathrm{II}(X, Z), Y\rangle
$$

where II denotes the second fundamental form of the immersion, $Y$ is the position vector field of $M$, and $\langle$,$\rangle denotes the Euclidean metric. The purpose of this$ section is to prove the following theorem.

THEOREM 2.1. If $S$ is a Codazzi tensor of type $(k, k)$ on $M$ then

$$
\int_{M}(n-k) \operatorname{trace} S+\operatorname{trace}(S * A) d V=0 .
$$

Two formulas for computing traces will be helpful in the proof. First, if $B \in \Gamma\left[\right.$ End $\left.\Lambda^{k}(T M)\right]$ is arbitrary and if $I \in \Gamma\left[\right.$ End $\left.\Lambda^{j}(T M)\right]$ is at each point of $M$ the identity, then trace $B * I=\left(\begin{array}{c}n-k \\ j\end{array}\right)$ trace $B$. Secondly, if $e_{1}, e_{2}, \ldots, e_{n}$ is a local orthonormal frame with $E=e_{1} \wedge e_{2} \wedge \cdots \wedge e_{n}$ then for $B \in \Gamma\left[\right.$ End $\left.\Lambda^{n-1}(T M)\right]$, $A \in \Gamma[$ End $(T M)]$, trace $B * A$ is equal to $\Sigma\left\langle A\left(e_{j}\right) \wedge B\left(E_{j}\right), e_{j} \wedge E_{j}\right\rangle$ where $E_{j}=$ $e_{1} \wedge e_{2} \wedge \cdots \wedge e_{j-1} \wedge e_{j+1} \wedge \cdots \wedge e_{n}$.

Proof of Theorem 2.1. Let $d V$ denote the volume element of $M$ and define an $n-1$ form $\alpha$ on $M$ by $\left.\alpha=Y^{\tan }\right\lrcorner d V$ (that is $\alpha\left(X_{1}, X_{2}, \ldots, X_{n-1}\right)$ is equal to $\left.d V\left(Y^{\tan }, X_{1}, X_{2}, \ldots, X_{n-1}\right)\right)$. It is routine to show that if $X$ is any $C^{\infty}$ vector field on $M$ then $\left.\nabla_{X} \alpha=[X+A(X)]\right\lrcorner d V$. Suppose first that $S$ is a Codazzi tensor of type $(n-1, n-1)$ and consider $\omega=\alpha \circ S$ as an $n-1$ form.

$$
\begin{aligned}
d \omega\left(e_{1}, \ldots, e_{n}\right) & =\sum(-1)^{j+1}\left(\nabla_{e_{j}} \omega\right)\left(E_{j}\right) \\
& =\sum(-1)^{j+1}\left(\nabla_{e_{j}} \alpha\right) \circ S\left(E_{j}\right)+\sum(-1)^{j+1} \alpha \circ\left(\nabla_{e_{j}} S\right)\left(E_{j}\right) .
\end{aligned}
$$

The second sum is zero since $S$ is a Codazzi tensor of type $(n-1, n-1)$. Thus

$$
\begin{aligned}
d \omega\left(e_{1}, \ldots, e_{n}\right) & \left.=\sum(-1)^{j+1}\left(e_{j}+A\left(e_{j}\right)\right)\right\lrcorner d V \circ S\left(E_{j}\right) \\
& =\sum(-1)^{j+1}\left\langle e_{j} \wedge S\left(E_{j}\right), E\right\rangle+\sum(-1)^{j+1}\left\langle A\left(e_{j}\right) \wedge S\left(E_{j}\right), E\right\rangle \\
& =\sum\left\langle e_{j} \wedge S\left(E_{j}\right), e_{j} \wedge E_{j}\right\rangle+\sum\left\langle A\left(e_{j}\right) \wedge S\left(E_{j}\right), e_{j} \wedge E_{j}\right\rangle \\
& =\operatorname{trace} S+\operatorname{trace} A * S .
\end{aligned}
$$

Since $0=\int_{M} d \omega$, the result follows in this case.

Suppose now that $S$ is a Codazzi tensor of type $(k, k)$ and let $I$ denote the identity element of $\Gamma$ [End $\Lambda^{n-k-1}(T M)$ ]. Since $I$ is parallel $S * I$ is a Codazzi tensor of type $(n-1, n-1)$ so that $0=\int_{M}$ trace $S * I+\operatorname{trace} S * A * I d V$. However trace $S * I=(n-k)$ trace $S$ and trace $S * A * I=\operatorname{trace} S * A$. 
Applications. (a) Let $T$ denote the shape operator of a compact hypersurface of Euclidean space with $\sigma_{k}$ the $k$ th elementary symmetric function of the eigenvalues of $T . S=T^{k}=T * T * \cdots * T$ ( $k$ times) is a Codazzi tensor of type $(k, k)$ with trace $S=k ! \sigma_{k}$. Furthermore $A=h T$ where $h$ denotes the support function of $M$ so that

$$
0=\int_{M}\left(\begin{array}{l}
n \\
k
\end{array}\right)^{-1} \sigma_{k}+\left(\begin{array}{c}
n \\
k+1
\end{array}\right)^{-1} \sigma_{k+1} h d V .
$$

These are the integral formulas of Hsiung [8].

(b) Suppose $N$ is a unit normal vector field on $M$ which is parallel in the normal bundle. Define a tensor $T$ of type $(1,1)$ by the formula $\langle\operatorname{II}(X, Z), N\rangle=$ $\langle T(X), Z\rangle . S=T^{k}$ is a Codazzi tensor of type $(k, k)$, and using Theorem 2.1 we recover the integral formulas of [3].

(c) Let $F$ be a differential quadratic form which is integrable in the sense of [4]. Define a tensor $S$ of type $(1,1)$ by the formula $F(X, Z)=\langle S(X), Z\rangle$. Then $S$ is a Codazzi tensor, and letting $k=1$ in Theorem 2.1 we recover Theorem 5 of [4]. In terms of $S$ this becomes

$$
0=\int_{M}(n-1) \operatorname{trace} S+(\operatorname{trace} S)(\operatorname{trace} A)-\operatorname{trace} A \circ S d V .
$$

(d) The curvature operators $R_{2 k} \in \Gamma\left[\right.$ End $\left.\Lambda^{2 k}(T M)\right]$ are defined by

$$
\left\langle R_{2}(X \wedge Y), Z \wedge W\right\rangle=\langle R(X, Y) Z, W\rangle
$$

and $R_{2 k}=R_{2} * R_{2} * \cdots * R_{2}$ ( $k$ times) where $R=\nabla_{[X, Y]}-\left[\nabla_{X}, \nabla_{Y}\right]$ denotes the curvature tensor. The second Bianchi identity implies $R_{2}$ is a Codazzi tensor of type $(2,2)$ and consequently $R_{2 k}$ is a Codazzi tensor of type $(2 k, 2 k)$. Therefore

$$
0=\int_{M}(n-2 k) \text { trace } R_{2 k}+\operatorname{trace} R_{2 k} * A d V .
$$

These formulas are equivalent to those of Proposition 1-b of [13].

A further application of Theorem 2.1 will be given in the next section.

3. Reducible submanifolds of Euclidean space. The most elementary endomorphisms of a vector space are the projections. If the vector space possesses an inner product we may restrict ourselves to the consideration of symmetric projections. Thus it is natural to ask under what conditions a symmetric tensor $P$, of type $(1,1)$ with $P \circ P=P$, satisfies the Codazzi equations.

Proposition 3.1. Let $P$ be a symmetric tensor of type $(1,1)$ on $M$ with $P \circ P=P$. The following are equivalent:

(a) $P$ is a Codazzi tensor.

(b) $P$ is parallel $(\nabla P \equiv 0)$.

(c) $P$ is projection onto a distribution $\pi$, with $\pi$ invariant under parallel translation along any path ( $\pi$ is parallel).

Proof. (a) implies (b). The 0 and 1 eigenspaces of $P$ at each point define orthogonal distributions $E_{0}$ and $E_{1}$ respectively on $M$. Let $X, Y, Z, W$ be $C^{\infty}$ 
vector fields on $M$ with $X, Z$ tangent to $E_{0}$ and $Y, W$ tangent to $E_{1} \cdot\left(\nabla_{X} P\right) Y$ $=\nabla_{X} Y-P\left(\nabla_{X} Y\right)$ which is equal to the component of $\nabla_{X} Y$ tangent to $E_{0}$. On the other hand $\left(\nabla_{Y} P\right) X=-P\left(\nabla_{Y} X\right)$ is tangent to $E_{1}$. Thus if $P$ satisfies the Codazzi equations then $\nabla_{X} Y$ is tangent to $E_{1}$ and $\nabla_{Y} X$ is tangent to $E_{0}$. Now $\left\langle\nabla_{X} Z, Y\right\rangle=$ $-\left\langle Z, \nabla_{X} Y\right\rangle=0$ and $\left\langle\nabla_{Y} W, X\right\rangle=-\left\langle W, \nabla_{Y} X\right\rangle=0$ and it easily follows that $P$ is parallel. (More generally if $S$ is a symmetric Codazzi tensor of type $(1,1)$ with precisely two distinct eigenvalues, both of which are constant, then $S$ is parallel.)

(b) implies (c). The eigenspaces of a parallel tensor of type $(1,1)$ are invariant under parallel translation along any path. Let $\pi$ be the eigenspace corresponding to eigenvalue 1 .

(c) implies (b). If $X$ and $Y$ are $C^{\infty}$ vector fields with $Y$ tangent to a parallel distribution then $\nabla_{X} Y$ is also tangent to the distribution. Denote by $\beta$ the distribution orthogonal to $\pi$. Then for $Y$ tangent to $\pi,\left(\nabla_{X} P\right) Y=\nabla_{X} Y-P\left(\nabla_{X} Y\right)$ $=0$. Similarly, since $\beta$ is parallel, $\left(\nabla_{X} P\right) Y=0$ for $Y$ tangent to $\beta$. Thus $P$ is parallel.

(b) implies (a). Obvious.

A manifold which possesses a nontrivial parallel distribution is called reducible. Theorem 2.1 and Proposition 3.1 motivate the consideration of compact reducible manifolds isometrically immersed into Euclidean space. However, before proceeding with the associated integral formulas we shall discuss briefly the codimension of such an immersion. First of all a compact reducible manifold of dimension two or greater cannot be isometrically immersed as a hypersurface of Euclidean space. This is because at every point of a reducible manifold there is a 2-plane with zero sectional curvature, while a compact hypersurface of Euclidean space possesses a point at which all sectional curvatures are positive.

In the case of higher codimension we have the following result.

Proposition 3.2. Let $M$ be a compact $n$ dimensional Riemannian manifold with $\pi_{1}, \pi_{2}, \ldots, \pi_{k}$ mutually orthogonal parallel distributions on $M$ such that $n_{i}=\operatorname{dim} \pi_{i}$ $\geqslant 2$ and $\sum n_{i}=n$. If $g: M \rightarrow \mathbf{R}^{n+k}$ is an isometric immersion then there exist compact manifolds $N_{i}, M_{i}$ of dimension $n_{i}$ and hypersurface immersions $g_{i}: N_{i} \rightarrow$ $\mathbf{R}^{n_{i}+1}, h_{i}: M_{i} \rightarrow \mathbf{R}^{n_{i}+1}$, such that the diagram below commutes:

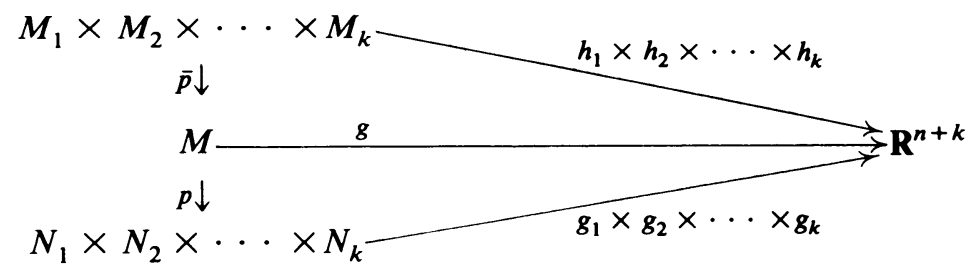

Furthermore $p$ and $\bar{p}$ are Riemannian covering maps with $\bar{p}_{*} T M_{i}=\pi_{i}$ and $p_{*} \pi_{i}=$ $T N_{i}$.

Proof. Assume without loss of generality that $k=2$. Let $(\tilde{M}, f: \tilde{M} \rightarrow M)$ denote the simply connected Riemannian covering space of $M$. The de Rham decomposition theorem implies that $\tilde{M}$ splits as a Riemannian product $L_{1} \times L_{2}$ with $f_{*}\left(T L_{i}\right)=\pi_{i}, i=1,2$. Let $I\left(L_{i}\right)$ denote the isometry group of $L_{i}$ and let 
$D=\left\{\Phi \in I\left(L_{1}\right) \times I\left(L_{2}\right) \mid f \circ \Phi=f\right\}$ denote the deck group of $M$. It is well known that $M$ is isometric to $\tilde{M} / D$. Let $\psi_{i}: D \rightarrow I\left(L_{i}\right)$ denote the natural projection and define subgroups $H_{i}$ of $D$ and $G_{i}$ of $I\left(L_{i}\right)$ by the short exact sequences

$$
\left(e_{1}, e_{2}\right) \rightarrow H_{2} \rightarrow D \stackrel{\psi_{1}}{\rightarrow} G_{1} \rightarrow e_{1}, \quad\left(e_{1}, e_{2}\right) \rightarrow H_{1} \rightarrow D \stackrel{\psi_{2}}{\rightarrow} G_{2} \rightarrow e_{2} .
$$

We then have a natural inclusion $D \rightarrow G_{1} \times G_{2}$.

Theorem 2 of [12] implies $g \circ f: \tilde{M} \rightarrow \mathbf{R}^{n+2}$ splits as a product immersion and consequently factors through the set $\tilde{M} / G_{1} \times G_{2}$. However, since $g \circ f$ is locally one-to-one it follows that $G_{1} \times G_{2}$ acts freely and properly discontinuously on $\tilde{M}$. Thus $\tilde{M} / G_{1} \times G_{2}$ may be given the structure of a Riemannian manifold isometric to $N_{1} \times N_{2}$ where $N_{i}=L_{i} / G_{i}$. The mapping $g \circ f: \tilde{M} \rightarrow \mathbf{R}^{n+2}$ factors through $N_{1} \times N_{2}$ as a product immersion $g_{1} \times g_{2}$. M is a covering space of $N_{1} \times N_{2}$ via $M \cong \tilde{M} / D \rightarrow^{p} \tilde{M} / G_{1} \times G_{2} \simeq N_{1} \times N_{2}$. Since $M$ is compact the index of $D$ in $G_{1} \times G_{2}$ is finite. Identifying $H_{i}$ with its projection into $G_{i}$ we have an injective mapping $G_{i} / H_{i} \rightarrow G_{1} \times G_{2} / D$ so that the index of $H_{i}$ in $G_{i}$ is finite. Thus the manifolds $M_{i}=L_{i} / H_{i}$ are compact and $g \circ f: \tilde{M} \rightarrow \mathbf{R}^{n+2}$ factors through $M_{1} \times$ $M_{2}$ as a product immersion $h_{1} \times h_{2} . M_{1} \times M_{2}$ covers $M$ via $M_{1} \times M_{2}=L_{1} / H_{1} \times$ $L_{2} / H_{2} \cong \tilde{M} / H_{1} H_{2} \rightarrow^{\bar{p}} \tilde{M} / D \cong M$.

The author wishes to thank Patrick Eberlein for pointing out the existence of the covering space $M_{1} \times M_{2} \times \cdots \times M_{k}$.

Corollary 3.3. If in addition to the hypotheses of Proposition $3.2, g$ is an embedding then $M$ splits as a Riemannian product and $g$ splits as a product embedding.

Now let $M$ be as in Theorem 2.1 with position vector field $Y$. If $\pi$ is a parallel distribution on $M$ then each leaf of $\pi$ is isometrically immersed into $\mathbf{R}^{N}$ by the restriction of $g: M \rightarrow \mathbf{R}^{N}$. Given $p \in M$ denote by $\eta(p)$ the mean curvature normal vector at $p$ for the corresponding immersed leaf of $\pi$.

LEMMA 3.4. $\int_{M}\langle\eta, Y\rangle d V=-\operatorname{vol} M$.

Proof. Let $P$ denote projection onto the distribution orthogonal to $\pi$. Since $P$ is parallel, $S=P^{k}=P * P * \cdots * P(k=n-\operatorname{dim} \pi$ times $)$ is a Codazzi tensor of type $(k, k)$. Trace $S=k !$, and trace $S * A=k !(\operatorname{dim} \pi)\langle\eta, Y\rangle$. The result now follows from Theorem 2.1.

Note. The conclusion of Lemma 3.4 actually holds under weaker assumptions. Suppose that $\pi$ is a nonparallel integrable distribution with totally geodesic leaves and the distribution orthogonal to $\pi$ is integrable with leaves which are minimal submanifolds of $M$. Let $P$ denote projection onto the distribution orthogonal to $\pi$. Although $P$ is not a Codazzi tensor, it is true that $S=P^{k}, k=n-\operatorname{dim} \pi$, is a Codazzi tensor of type $(k, k)$ and the argument of Lemma 3.4 may still be applied.

Let $\lambda_{1}$ denote the first eigenvalue of the Laplacian on $M$. With the hypotheses of Lemma 3.4 we have

THeOREM 3.5. $\int_{M}|\eta|^{2} d V \geqslant\left(\lambda_{1}\right.$ vol $\left.M\right) / n$ with equality iff both $M$ and the leaves of $\pi$ are minimally immersed into a sphere of radius $R=\left(n / \lambda_{1}\right)^{1 / 2}$. 
Proof. Assume without loss of generality that $\int_{M} Y d V=\overrightarrow{0}$. The CauchySchwarz inequalities for $\langle$,$\rangle and for integrals imply \left(\int_{M}|\eta|^{2} d V\right)\left(\int_{M}|Y|^{2} d V\right) \geqslant$ $(\operatorname{vol} M)^{2}$ with equality iff $\eta=c Y$ for some constant $c$. Reilly [13] showed that $\int_{M}|Y|^{2} d V \leqslant(n$ vol $M) / \lambda_{1}$ with equality iff $M$ is minimally immersed into a sphere about the origin of radius $R=\left(n / \lambda_{1}\right)^{1 / 2}$. Therefore if $\int_{M}|\eta|^{2} d V=\left(\lambda_{1}\right.$ vol $\left.M\right) / n$ then (1) $M$ is minimally immersed into a sphere of radius $R=\left(n / \lambda_{1}\right)^{1 / 2}$ and (2) $\eta=c Y$ which implies each leaf of $\pi$ is a minimal submanifold of the given sphere. On the other hand if each leaf of $\pi$ is minimally immersed in a sphere of radius $R=\left(n / \lambda_{1}\right)^{1 / 2}$ then $\eta=-\left(\lambda_{1} / n\right)^{1 / 2} N$ where $N$ denotes the outward normal to the sphere and therefore $\int_{M}|\eta|^{2} d V=\left(\lambda_{1}\right.$ vol $\left.M\right) / n$.

Note. (i) Theorem 3.5 extends Reilly's Theorem A [13] for the case $r=1$ to reducible submanifolds.

(ii) $R=\left(n / \lambda_{1}\right)^{1 / 2}$ is significant in that $M$ cannot be minimally immersed into a sphere of larger radius.

COROLlaRY 3.6. Let $M$ be a circle of radius $r$, isometrically immersed into $\mathbf{R}^{N}$. If $k$ denotes the curvature function then $\int_{M} k^{2} d V \geqslant 2 \pi / r$ with equality iff $M$ is embedded as a circle.

Proof. If $\eta$ denotes the mean curvature normal vector field of $M$ then $|\eta|^{2}=k^{2}$. It is known that $\lambda_{1}=1 / r^{2}$. The result now follows from Theorem 3.5.

Denote by the triple $(M, g, \pi)$ a manifold $M$ and immersion $g: M \rightarrow \mathbf{R}^{N}$ satisfying the hypotheses of Theorem 3.5, with $\pi$ a parallel distribution on $M$. Let $S=\{(M, g, \pi) \mid$ equality holds in Theorem 3.5 $\}$. Note that $(M, g, \pi) \in S$ implies $(M, g, T M) \in S$ where $T M$ denotes the distribution which at each point $x$ is just $T_{x} M$.

Proposition 3.7. Let $\pi_{1}, \pi_{2}$ be orthogonal nonzero parallel distributions on $M$ with $\left(M, g, \pi_{1}\right) \in S$. Then $\left(M, g, \pi_{1}+\pi_{2}\right) \in S$ iff $\left(M, g, \pi_{2}\right) \in S$. In particular if $0<$ $\operatorname{dim} \pi<\operatorname{dim} M$ then $(M, g, \pi) \in S$ iff $(M, g, \beta) \in S$ where $\beta$ denotes the parallel distribution orthogonal to $\pi$.

Proof. If $\eta_{i}$ denotes the mean curvature normal vector field to the leaves of $\pi_{i}$ and $\eta$ denotes the mean curvature normal vector field to the leaves of $\pi_{1}+\pi_{2}$ then $\eta=\left(k_{1} \eta_{1}+k_{2} \eta_{2}\right) /\left(k_{1}+k_{2}\right)$ where $k_{i}=\operatorname{dim} \pi_{i} \cdot\left(M, g, \pi_{1}\right) \in S$ implies $\eta_{1}=$ $-\left(\lambda_{1} / n\right)^{1 / 2} N$, where $N$ denotes the outward normal to the sphere. $\left(M, g, \pi_{1}+\pi_{2}\right)$ $\in S$ iff $\eta=-\left(\lambda_{1} / n\right)^{1 / 2} N$. But this happens iff $\eta_{2}=-\left(\lambda_{1} / n\right)^{1 / 2} N$ iff $\left(M, g, \pi_{2}\right) \in S$.

A natural way of constructing manifolds with parallel distributions is by taking Riemannian products. Suppose $M_{1}, M_{2}$ are compact, oriented connected Riemannian manifolds without boundary. Let $M=M_{1} \times M_{2}$ be the Riemannian product of $M_{1}$ and $M_{2}$ with $\pi$ a distribution defined by $\pi(x, y)=T_{y} M_{2}$. Then $\pi$ is a parallel distribution on $M$ and we write for simplicity $\pi=T M_{2}$. However no element of $S$ can be constructed in this way. If fact it will be shown that if $\pi$ is a parallel distribution on a product manifold $M_{1} \times M_{2}$, and $g: M_{1} \times M_{2} \rightarrow \mathbf{R}^{N}$ is an isometric immersion with $\left(M_{1} \times M_{2}, g, \pi\right) \in S$ then (a) $\pi$ is tangent to neither $M_{1}$ nor 
$M_{2}$, (b) $g$ splits as a product immersion $g=g_{1} \times g_{2}$, (c) $\left(M_{i}, g_{i}, T M_{i}\right) \in S$, and (d) $\lambda_{1}\left(M_{1}\right)=\lambda_{1}\left(M_{2}\right)$ where $\lambda_{1}\left(M_{i}\right)$ denotes the first eigenvalue of the Laplacian on $M_{i}$.

Proposition 3.8. Suppose $(M, g, \pi) \in S$ and $M_{1} \times M_{2}$ is a compact, oriented Riemannian covering space of $M$ with the lift $\tilde{\pi}$ of $\pi$ to $M_{1} \times M_{2}$ tangent to $M_{2}$. Then $\lambda_{1}\left(M_{1} \times M_{2}\right) \leqslant\left(n_{2} / n\right) \lambda_{1}(M), n_{i}=\operatorname{dim} M_{i}$.

Proof. Since $\lambda_{1}\left(M_{1} \times M_{2}\right)=\min \left(\lambda_{1}\left(M_{1}\right), \lambda_{1}\left(M_{2}\right)\right)$ it suffices to show that $\lambda_{1}\left(M_{2}\right) \leqslant\left(n_{2} / n\right) \lambda_{1}(M)$. Let $\tilde{\eta}$ denote the mean curvature normal vector field to the leaves of $\tilde{\pi}$ immersed into Euclidean space by the composition $M_{1} \times M_{2} \rightarrow M$ $\rightarrow^{g} \mathbf{R}^{N}$. If $(M, g, \pi) \in S$ then $\int_{M}|\eta|^{2} d V=\left(\lambda_{1}\right.$ vol $\left.M\right) / n$ which implies

$$
\int_{M_{1} \times M_{2}}|\tilde{\eta}|^{2} d V_{2} d V_{1}=\frac{\lambda_{1}(M) \operatorname{vol}\left(M_{1} \times M_{2}\right)}{n} \text {. }
$$

Let $h$ denote the composition $M_{1} \times M_{2} \rightarrow M \rightarrow^{8} \mathbf{R}^{N}$. If we fix a point $x \in M_{1}$ then $h(x):, M_{2} \rightarrow \mathbf{R}^{N}$ is an isometric immersion of $M_{2}$ into Euclidean $N$ space. Furthermore it can be shown that the mean curvature normal vector to the leaf of $\tilde{\pi}$ at $y \in M_{2}$ is just $\tilde{\eta}(x, y)$. Therefore

$$
\int_{M_{1} \times M_{2}}|\tilde{\eta}|^{2} d V_{2} d V_{1}=\int_{M_{1}}\left[\int_{M_{2}}\left|\tilde{\eta}\left(x_{1}, x_{2}\right)\right|^{2} d V_{2}\right] d V_{1} \geqslant \frac{\lambda_{1}\left(M_{2}\right) \text { vol } M_{1} \times M_{2}}{n_{2}}
$$

by Theorem 3.5. Thus $\left(\lambda_{1}(M)\right.$ vol $\left.M_{1} \times M_{2}\right) / n>\left(\lambda_{1}\left(M_{2}\right)\right.$ vol $\left.M_{1} \times M_{2}\right) / n_{2}$ and the result follows.

Letting $M=M_{1} \times M_{2}$ yields

Corollary 3.9. $\left(M_{1} \times M_{2}, g, \pi\right) \notin S$ for any $\pi$ tangent to $M_{2}$. In particular $(M, g, \pi) \notin S$ for $M$ simply connected and $\operatorname{dim} \pi<\operatorname{dim} M$.

Proposition 3.10. Given $\pi_{i}$ a parallel distribution on $M_{i}$ with $k_{i}=\operatorname{dim} \pi_{i}$ then $\left(M_{1} \times M_{2}, g, \pi_{1}+\pi_{2}\right) \in S$ iff:

(a) $g$ splits as a product immersion $g=g_{1} \times g_{2}$;

(b) $\left(M_{i}, g_{i}, \pi_{i}\right) \in S, i=1,2$;

(c) $\lambda_{1}\left(M_{1}\right)=\lambda_{1}\left(M_{2}\right)$

(d) $k_{1} n_{2}=k_{2} n_{1}, n_{i}=\operatorname{dim} M_{i}$.

Proof. A routine argument shows that

$$
\frac{k_{1}^{2}}{\left(k_{1}+k_{2}\right)^{2} n_{1}}+\frac{k_{2}^{2}}{\left(k_{1}+k_{2}\right)^{2} n_{2}} \geqslant \frac{1}{n_{1}+n_{2}}
$$

with equality iff $k_{1} n_{2}=k_{2} n_{1}$. Let $g: M_{1} \times M_{2} \rightarrow \mathbf{R}^{N}$ be an isometric immersion with $\eta_{i}$ the mean curvature normal vector field to the leaves of $\pi_{i}$ and $\eta=$ $\left(k_{1} \eta_{1}+k_{2} \eta_{2}\right) /\left(k_{1}+k_{2}\right)$ the mean curvature normal vector field to the leaves of $\pi_{1}+\pi_{2}$.

$$
|\eta|^{2}=\frac{k_{1}^{2}}{\left(k_{1}+k_{2}\right)^{2}}\left|\eta_{1}\right|^{2}+\frac{k_{2}^{2}}{\left(k_{1}+k_{2}\right)^{2}}\left|\eta_{2}\right|^{2}+\frac{2 k_{1} k_{2}}{\left(k_{1}+k_{2}\right)^{2}}\left\langle\eta_{1}, \eta_{2}\right\rangle .
$$


As in the proof of Proposition 3.8

$$
\int_{M_{1} \times M_{2}}\left|\eta_{2}\right|^{2} d V_{2} d V_{1}=\int_{M_{1}}\left[\int_{M_{2}}\left|\eta_{2}\right|^{2} d V_{2}\right] d V_{1} \geqslant\left(\lambda_{1}\left(M_{2}\right) \text { vol } M_{1} \times M_{2}\right) / n_{2}
$$

Similarly $\int_{M_{1} \times M_{2}}\left|\eta_{1}\right|^{2} d V_{2} d V_{1} \geqslant\left(\lambda_{1}\left(M_{1}\right)\right.$ vol $\left.M_{1} \times M_{2}\right) / n_{1}$. The Gauss curvature equations imply

$$
\frac{2 k_{1} k_{2}}{\left(k_{1}+k_{2}\right)^{2}} \int_{M_{1} \times M_{2}}\left\langle\eta_{1}, \eta_{2}\right\rangle d V_{2} d V_{1}=C \geqslant 0
$$

where $C=0$ iff $\left\langle\eta_{1}, \eta_{2}\right\rangle \equiv 0$. If we let $A=k_{1}^{2} /\left(k_{1}+k_{2}\right)^{2} n_{1}, B=k_{2}^{2} /\left(k_{1}+k_{2}\right)^{2} n_{2}$, then we have a string of inequalities

$$
\begin{aligned}
& \int_{M_{1} \times M_{2}}|\eta|^{2} d V_{2} d V_{1} \geqslant A \lambda_{1}\left(M_{1}\right) \text { vol } M_{1} \times M_{2}+B \lambda_{1}\left(M_{2}\right) \text { vol } M_{1} \times M_{2}+C \\
& \geqslant A \lambda_{1}\left(M_{1} \times M_{2}\right) \text { vol } M_{1} \times M_{2}+B \lambda_{1}\left(M_{1} \times M_{2}\right) \text { vol } M_{1} \times M_{2}+C \\
& \geqslant \frac{\lambda_{1}\left(M_{1} \times M_{2}\right) \operatorname{vol} M_{1} \times M_{2}}{n_{1}+n_{2}}+C \geqslant \frac{\lambda_{1}\left(M_{1} \times M_{2}\right) \operatorname{vol} M_{1} \times M_{2}}{n_{1}+n_{2}} .
\end{aligned}
$$

Note in particular that if $\left(M_{1} \times M_{2}, g, T M_{1}+T M_{2}\right)$ is an element of $S$ then $C=0$, which implies the mean curvature normal vector fields of $M_{1}$ and $M_{2}$ are everywhere orthogonal. But this implies $g$ splits as a product immersion $g=g_{1} \times$ $g_{2}$ [5]. Now if $\left(M_{1} \times M_{2}, g, \pi_{1}+\pi_{2}\right) \in S$ then $\left(M_{1} \times M_{2}, g, T M_{1}+T M_{2}\right) \in S$ so that $g$ indeed splits as a product immersion. Furthermore all the inequalities above must be in fact equalities, from which conditions (b)-(d) follow. On the other hand if (a)-(d) hold then $\left(M_{1} \times M_{2}, g, \pi_{1}+\pi_{2}\right) \in S$.

Corollary 3.11. If $\left(M_{1} \times M_{2}, g, \pi\right) \in S$ then

(a) $g$ splits as a product immersion $g=g_{1} \times g_{2}$;

(b) $\left(M_{i}, g_{i}, T M_{i}\right) \in S, i=1,2$;

(c) $\lambda_{1}\left(M_{1}\right)=\lambda_{1}\left(M_{2}\right)$.

Proof. If $\left(M_{1} \times M_{2}, g, \pi\right) \in S$ then $\left(M_{1} \times M_{2}, g, T M_{1}+T M_{2}\right) \in S$ and the result follows from Proposition 3.10.

EXAMPle 3.1. Suppose $g: S^{n} \times S^{n} \rightarrow S^{N}\left(2^{1 / 2}\right)$ is a minimal immersion where $S^{N}\left(2^{1 / 2}\right)$ denotes an $N$ sphere of radius $2^{1 / 2}$. Since $\lambda_{1}\left(S^{n} \times S^{n}\right)=n$ it follows from Theorem 3.5 that $\left(S^{n} \times S^{n}, g, T S^{n}+T S^{n}\right) \in S$. Thus $g$ splits as a product immersion $g=g_{1} \times g_{2}$, where $g_{i}: S^{n} \rightarrow S^{N_{i}}$ is a minimal immersion. It follows from the Gauss curvature equations that $g_{i}$ embeds $S^{n}$ as a great $n$ sphere in $S^{N_{i}}$.

Now suppose $M=M_{1} \times M_{2}$ is a Riemannian product with $\pi$ a parallel distribution on $M$. Let $P$ be projection onto $\pi$ and denote by $P_{i}$ projection onto $T M_{i}$. The image of $P_{i} \circ P$ is a parallel distribution on $M$, tangent to $M_{i}$. Define the function $\left|P_{i} \circ P\right|$ by the formula $\left|P_{i} \circ P\right|(p)=\sup _{|v|=1}\left|P_{i}(P(v))\right|$ where $v \in T_{p} M$. It is easy to check that $P_{1} \circ P\left(T_{(x, y)} M\right)$ is independent of $y$ and defines a parallel distribution $\pi_{1}$ on $M_{1}$. Similarly $\pi_{2}(y)=P_{2} \circ P\left(T_{(x, y)} M\right)$ defines a parallel distribution on $M_{2}$.

If $\operatorname{dim} \pi=1$ we can strengthen Corollary 3.11 with the following result. (The proof, similar to that of Proposition 3.10, will be omitted.) 
Proposition 3.12. If $\operatorname{dim} \pi=1$ then $\left(M_{1} \times M_{2}, g, \pi\right) \in S$ iff

(a) $g$ splits as a product immersion $g=g_{1} \times g_{2}$;

(b) $\left(M_{i}, g_{i}, \pi_{i}\right) \in S$, where $\pi_{i}$ is defined as above;

(c) $\lambda_{1}\left(M_{1}\right)=\lambda_{1}\left(M_{2}\right)$;

(d) $\left|P_{i} \circ P\right|=\left(n_{i} / n\right)^{1 / 2}, n_{i}=\operatorname{dim} M_{i}, n=n_{1}+n_{2}$.

EXAMPLE 3.2. Let $M=R^{2} / Z^{2}$ with $g: M \rightarrow \mathbf{R}^{4}$ the standard embedding (considering $M$ as a product of two circles). Let $\pi$ be the parallel distribution on $M$ generated by the lines of slope 1 in $\mathbf{R}^{2}$. Proposition 3.12 then implies $(M, g, \pi) \in S$. As the next result shows this is the typical example of a reducible surface in $S$.

TheOREM 3.13. If $(M, g, \pi) \in S$ with $\operatorname{dim} M=2$ and $\operatorname{dim} \pi=1$ then $M$ is isometric to the flat torus $\mathbf{R}^{2} / \alpha\left(\mathbf{Z}^{2}\right), \alpha=2 \pi /\left(\lambda_{1}\right)^{1 / 2}$, and $g$ splits as a product embedding of $M$ into a 4 dimensional flat of $\mathbf{R}^{N}$. Furthermore $\pi$ is generated by the lines of slope 1 or -1 in $\mathbf{R}^{2}$.

Before proving Theorem 3.13 we recall some facts about the Laplacian on a flat torus. A subgroup $\Gamma$ of $\left(\mathbf{R}^{2},+\right)$ is called a lattice provided $\Gamma$ is generated by two linearly independent vectors of $\mathbf{R}^{2}$. Given a lattice $\Gamma$, let $\Gamma^{*}=\left\{v \in \mathbf{R}^{2} \mid\langle v, w\rangle\right.$ is an integer for all $w \in \Gamma\}$. Then $\Gamma^{*}$ is also a lattice, called the dual lattice of $\Gamma$, and $\left(\Gamma^{*}\right)^{*}=\Gamma$. The eigenvalues of the Laplacian on the flat torus $\mathbf{R}^{2} / \Gamma$ are given by $\left\{\lambda=4 \pi^{2}|v|^{2} \mid v \in \Gamma^{*}\right\}[1]$. In particular $\lambda_{1}=4 \pi^{2}\left|v_{0}\right|^{2}$ where $v_{0}$ is a nonzero vector in $\Gamma^{*}$ of minimum length. Furthermore if $w_{0} \in \Gamma^{*}$ is orthogonal to $v_{0}$ and of equal length then $\left\{v_{0}, w_{0}\right\}$ generate $\Gamma^{*}$.

Proof of Theorem 3.13. $\operatorname{dim} M=2$ and $\operatorname{dim} \pi=1 \mathrm{imply} M$ is flat. Since $M$ is compact and oriented, $M$ is isometric to $\mathbf{R}^{2} / \Gamma$ for some lattice $\Gamma$ and we write for simplicity $M=\mathbf{R}^{2} / \Gamma$. Let $p: \mathbf{R}^{2} \rightarrow M$ denote the Riemannian covering map with $\beta$ the lift of $\pi$ to $\mathbf{R}^{2}$. Assume without loss of generality that $\beta$ is generated by the vector field $e=\left(\frac{1}{2}\right)^{1 / 2} \partial / \partial x+\left(\frac{1}{2}\right)^{1 / 2} \partial / \partial y$. If $(M, g, \pi) \in S$ then $\mathbf{R}^{2}$ and the leaves of $\beta$ are minimally immersed into a sphere of radius $R=\left(2 / \lambda_{1}\right)^{1 / 2}$ by $g \circ p: \mathbf{R}^{2} \rightarrow$ $\mathbf{R}^{N}$. A routine computation shows that the mean curvature normal vector fields of $\mathbf{R}^{2}$ and the leaves of $\beta$ must be equal at corresponding points and therefore

$$
\mathrm{II}(e, e)=\frac{1}{2}(\mathrm{II}(\partial / \partial x, \partial / \partial x)+\mathrm{II}(\partial / \partial y, \partial / \partial y)) .
$$

By our choice of $e$ we must have $\mathrm{II}(\partial / \partial x, \partial / \partial y) \equiv 0$ which implies $g \circ p$ splits as a product immersion [12].

Let $G$ be the subgroup of $\left(\mathbf{R}^{2},+\right)$ generated by $\{(x, 0),(y, 0) \mid(x, y) \in \Gamma\}$. Clearly $\Gamma$ is a subgroup of $G$. Since $g \circ p$ splits as a product immersion and factors through $M=\mathbf{R}^{2} / \Gamma, g \circ p$ factors through $X=\mathbf{R}^{2} / G$. Since $g \circ p$ is locally $1-1$ it follows that $G$ is a lattice with one generator in each coordinate direction and $X$ splits as a product of circles, $X_{1} \times X_{2}$. We then have the following commutative diagram:

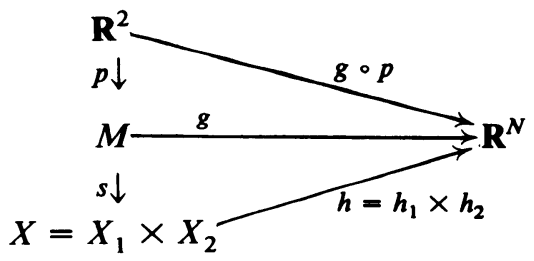


The distribution $\beta$ on $\mathbf{R}^{2}$ descends to a parallel distribution $\tilde{\pi}$ on $X$ with $s_{*} \pi=\tilde{\pi}$. Let $\tilde{\eta}$ denote the mean curvature normal vector field to the leaves of $\tilde{\pi}$ immersed into $\mathbf{R}^{N}$ by the restriction of $h: X \rightarrow \mathbf{R}^{N}$. If $(M, g, \pi) \in S$ and $s: M \rightarrow X$ is an $r$-fold covering then

$$
\frac{\lambda_{1}(M) \operatorname{vol} M}{2}=\int_{M}|\eta|^{2} d V=r \int_{X}|\tilde{\eta}|^{2} d X \geqslant \frac{r \lambda_{1}(X) \operatorname{vol} X}{2}=\frac{\lambda_{1}(X) \operatorname{vol} M}{2} .
$$

Eigenfunctions of the Laplacian on $X$ lift under $s$ to eigenfunctions on $M$ and thus $\lambda_{1}(M) \leqslant \lambda_{1}(X)$. Therefore we must have (1) $\lambda_{1}(M)=\lambda_{1}(X)$ and (2) $(X, h, \tilde{\pi}) \in S$. Corollaries 3.6 and 3.11 imply $G$ is a square lattice, $G=\alpha \mathbf{Z}^{2}, \alpha=2 \pi /\left(\lambda_{1}(M)\right)^{1 / 2}$ and $h$ is a product embedding of $X$ into a 4 dimensional flat of $\mathbf{R}^{N}$. $G^{*}$ is a square lattice contained in $\Gamma^{*}$ and since $\lambda_{1}(M)=\lambda_{1}(X)$ it follows that $G^{*}=\Gamma^{*}$ and thus $G=\Gamma$.

4. Parallel tensors of type $(1,1)$. An important class of Codazzi tensors is the space of parallel tensors of type $(1,1)$ on $M$. Here we study this space in terms of the de Rham decomposition of $M$ and derive some related results concerning Kaehler manifolds.

Definition 4.1. A tensor $J$ of type $(1,1)$ is a Kaehler structure for $M$ provided

(a) $J \circ J=-I$;

(b) $\langle J(X), J(Y)\rangle=\langle X, Y\rangle$ for all $C^{\infty}$ vector fields $X, Y$;

(c) $\nabla_{X} J=0$ for all $C^{\infty}$ vector fields $X$.

Proposition 4.1. If $M$ is irreducible and $A$ is a parallel tensor of type $(1,1)$ on $M$ then either $A=\alpha I$ or $A=\alpha I+\beta J, \alpha, \beta \in \mathbf{R}, J$ a Kaehler structure (depending on A) on $M$.

Proof. Assume first that $A$ is skew-symmetric. Since $A$ is parallel, $A^{t}$ is parallel and thus $A^{t} \circ A$ is parallel. If $A$ is nonzero then $A^{t} \circ A$ is symmetric positive definite and the eigenspaces of $A^{t} \circ A$ define parallel distributions on $M$. Since $M$ is irreducible $A^{t} \circ A=c I$, where $c$ is a positive real number. It is easy to check that $J=c^{-1 / 2} A$ is a Kaehler structure on $M$. For general $A$, write $A$ as the sum of a symmetric plus a skew-symmetric tensor of type $(1,1)$.

The space $Z$ of parallel tensors of type $(1,1)$ on $M$ is a real algebra under the operations of addition and composition. The next result (which is also proved in [10]) determines the possible isomorphism classes of $Z$ when $M$ is irreducible.

Proposition 4.2. If $M$ is irreducible then $Z$ is isomorphic to one of the following: (i) the real numbers $\mathbf{R}$, (ii) the complex numbers $\mathbf{C}$, (iii) the quaternions $\mathbf{H}$.

Proof. It suffices to show $Z$ is a finite dimensional real division algebra for then the result follows from the well-known classification theorem of Frobenius. If $A \in Z$ then $\operatorname{Ker} A$ defines a parallel distribution on $M$. Since $M$ is irreducible either $\operatorname{Ker} A=0$ or $\operatorname{Ker} A=T_{p} M$ for all $p \in M$. Consequently if $A \neq 0$ then $A$ is invertible, and since $0=\nabla_{X} I=\nabla_{X}\left(A \circ A^{-1}\right)=\left(\nabla_{X} A\right) \circ A^{-1}+A \circ\left(\nabla_{X} A^{-1}\right)=$ $A \circ\left(\nabla_{X} A^{-1}\right)$ it follows that $A^{-1}$ is an element of $Z$. Therefore $Z$ is a real division algebra. If we fix a point $x \in M$, then the linear mapping $L: Z \rightarrow$ End $T_{x} M$ defined by $L(A)=A_{x}$ is injective and thus $Z$ is finite dimensional. 
If $Z$ is isomorphic to $\mathbf{C}$ or $\mathbf{H}$ then using Proposition 4.1 it is easy to show that the purely imaginary elements of norm 1 correspond to the Kaehler structures of $M$. If $Z$ is isomorphic to $\mathbf{H}$ then, at each point $p \in M, T_{p} M$ becomes a module over $H$ and thus $n=\operatorname{dim} M$ is divisible by 4 . Furthermore there must exist Kaehler structures $J$ and $K$ on $M$ with $J \circ K=-K \circ J$. As A. Gray [7] has pointed out, this implies $M$ is Ricci flat. Consequently if either $n$ is not divisible by 4 or $M$ is not Ricci flat then the space $Z$ is at most 2 dimensional (for $M$ irreducible).

Suppose now that $M$ is a Kaehler manifold with Kaehler structure $J$ and Kaehler two form $\Phi$ defined by $\Phi(X, Y)=\langle X, J(Y)\rangle$. If $\operatorname{dim} Z=2$ and if $D$ is a subgroup of isometries of $M$ then $f_{*}^{-1} \circ J \circ f_{*}= \pm J$ for all $f \in D$. Consequently $f^{*} \Phi^{2 k}=$ $\Phi^{2 k}, k=1,2, \ldots$ Furthermore we may define a group homomorphism, $\psi: D \rightarrow$ $\{-1,+1\}$, by $f_{*}^{-1} \circ J \circ f_{*}=\psi(f) J$. Since the subgroup $D^{J}$ of $D$ leaving $J$ invariant is equal to $\operatorname{Ker} \psi$, either $D^{J}=D$ or the index of $D^{J}$ in $D$ is 2 .

Proposition 4.3. Let $M$ be an $n$ dimensional irreducible Riemannian manifold which is covered by a Kaehler manifold.

(a) If $M$ is compact with finite fundamental group and if either $n$ is not divisible by 4 or $M$ is not Ricci flat then the $4 k$ th Betti numbers of $M$ are nonzero.

(b) If $n$ is not divisible by 4 and $M$ is oriented then $M$ is itself Kaehler.

(c) If $n$ is divisible by 4 and $M$ is not Ricci flat then $M$ is oriented and is either Kaehler or is doubly covered by a Kaehler manifold.

Proof. Let $\pi: \tilde{M} \rightarrow M$ denote the simply connected cover of $M$ with $D(\simeq$ $\left.\pi_{1}(M)\right)$ the deck group of $M$. If $M$ is covered by a Kaehler manifold $N$ we may form the tower $\tilde{M} \rightarrow N \rightarrow M$ and lift the Kaehler structure of $N$ to a Kaehler structure $J$ on $\tilde{M}$. Let $\Phi$ denote the associated Kaehler two form on $\tilde{M}$.

(a) $\pi_{1}(M)$ finite implies $\tilde{M}$ is compact and that $\Phi^{2 k}$ is a closed nonexact $4 k$ form on $\tilde{M}$ for $4 \leqslant 4 k \leqslant n$. Since $f^{*} \Phi^{2 k}=\Phi^{2 k}$ for all $f \in D, \Phi^{2 k}$ descends to a closed nonexact $4 k$ form on $M$ and thus the $4 k$ th Betti numbers of $M$ are nonzero.

(b) Choose a volume form $d V$ on $M$ such that $\pi^{*} d V=\Phi^{n / 2}$. With $\psi$ as above, $f^{*} \Phi^{n / 2}=\psi(f) \Phi^{n / 2}$. On the other hand, for each $f$ in $D$,

$$
f^{*} \Phi^{n / 2}=f^{*} \circ \pi^{*} d V=(\pi \circ f)^{*} d V=\pi^{*} d V=\Phi^{n / 2}
$$

so that $D^{J}=D$ and $J$ descends to a Kaehler structure on $M$.

(c) If $n=4 k$ then $f^{*} \Phi^{2 k}=\Phi^{2 k}$ for every isometry $f$ of $\tilde{M}$. Thus the volume form $\Phi^{2 k}$ on $\tilde{M}$ descends to a volume element on $M$ and $M$ is oriented. Since either $D^{J}=D$ or the index of $D^{J}$ in $D$ is 2 it follows that either $M$ is Kaehler or is doubly covered by a Kaehler manifold.

COROLlaRY 4.4. Let $M$ be a compact irreducible Riemannian manifold which is covered by a Kaehler manifold. If the Ricci tensor of $M$ is positive definite then either $M$ is simply connected or $\pi_{1}(M)=\mathbf{Z} /(2)$.

Proof. Myers' theorem (for the Ricci tensor) implies $\tilde{M}$ is compact and thus any Riemannian cover of $M$ is also compact. It follows from Proposition 4.3 that either $M$ is Kaehler or is doubly covered by a Kaehler manifold. By a theorem due to 
Kobayashi [9], a compact Kaehler manifold with positive definite Ricci tensor must be simply connected and the result follows.

The next result shows that parallel tensors of type $(1,1)$ respect the de Rham decomposition.

Proposition 4.5. Let $M$ be complete and simply connected with de Rham decomposition $M=M_{0} \times M_{1} \times \cdots \times M_{k}$ and let $A$ be a parallel tensor of type $(1,1)$ on M. Then $A=A_{0} \times A_{1} \times \cdots \times A_{k}$ where $A_{j}$ is a parallel tensor of type $(1,1)$ on $M_{j}$.

Proof. If we fix a point $x=\left(x_{0}, \ldots, x_{k}\right)$ in $M$ then $T_{x} M$ may be written as an orthogonal direct sum, $T_{x} M=\sum T_{x}^{i}$ where $T_{x}^{i}=T_{x_{i}} M_{i}$. Furthermore

(a) The holonomy group at $x, \Psi(x)$, is the direct product $\psi_{0}(x) \times \cdots \times \psi_{k}(x)$ where $\psi_{i}(x)$ acts as the identity on $T_{x}^{j}$ for $i \neq j$ and $\psi_{0}(x)$ consists of the identity alone. If $v \in T_{x}^{i}$ for $i \neq 0$ then there exists $\alpha \in \psi_{i}(x)$ with $\alpha(v) \neq v$.

(b) If $S_{x}$ is a subspace of $T_{x} M$ which is invariant under $\Psi(x)$ then, for each $i=1,2, \ldots, k$, either $S_{x}$ is orthogonal to $T_{x}^{i}$ or $S_{x}$ contains $T_{x}^{i}$. (Proofs of these results may be found in [11].)

We first show that $A_{x}$ carries $T_{x}^{i}$ into $T_{x}^{i}$ for all $i$. $A$ parallel implies $A$ commutes with each element of $\Psi(x)$. If $v \in T_{x}^{0}$ and $\alpha \in \Psi(x)$ then $\alpha\left(A_{x}(v)\right)=A_{x}(\alpha(v))=$ $A_{x}(v)$ so that $A_{x}: T_{x}^{0} \rightarrow T_{x}^{0}$. Now $A$ parallel implies $\operatorname{Ker} A_{x}$ is invariant under $\Psi(x)$ so that we may write $\operatorname{Ker} A_{x}=V_{0}+T_{x}^{i_{1}}+\cdots+T_{x}^{i_{s}}$ where $V_{0}$ is some subspace of $T_{x}^{0}$ and + denotes an orthogonal direct sum. Clearly $A_{x}: T_{x}^{i_{i}} \rightarrow T_{x}^{i_{i}}, i=1, \ldots, s$. Consider $T_{x}^{j}$ where $j \notin\left\{0, i_{1}, \ldots, i_{s}\right\}$. If $A_{x}\left(T_{x}^{j}\right)$ is not orthogonal to $T_{x}^{j}$ then $A_{x}\left(T_{x}^{j}\right)=T_{x}^{j}$ since $A_{x}\left(T_{x}^{j}\right)$ is invariant under $\Psi(x)$. Suppose $A_{x}\left(T_{x}^{j}\right)$ is orthogonal to $T_{x}^{j}$. Since $j \neq 0$ if $v \in T_{x}^{j}$ then there exists $\alpha \in \Psi(x)$ such that $v \neq \alpha(v)$ and $\alpha$ acts as the identity on the orthogonal complement to $T_{x}^{j}$. But then $A_{x}(v)=\alpha A_{x}(v)=$ $A_{x}(\alpha v)$ which contradicts the fact that $A_{x}$ is 1-1 on $T_{x}^{j}$. Thus $A_{x}: T_{x}^{i} \rightarrow T_{x}^{i}$, $i=0,1, \ldots, k$.

Let $\gamma$ be a path in $M_{1} \times M_{2} \times \cdots \times M_{k}$ with initial point $\left(x_{1}, x_{2}, \ldots, x_{k}\right)$ and final point $\left(x_{1}^{\prime}, x_{2}^{\prime}, \ldots, x_{k}^{\prime}\right)$. Given $x_{0} \in M_{0}, \gamma$ induces a path in $M$ with initial point $\left(x_{0}, x_{1}, \ldots, x_{k}\right)$ and final point $\left(x_{0}, x_{1}^{\prime}, \ldots, x_{k}^{\prime}\right)$. Let $P_{\gamma}$ denote parallel translation along this path, and $P_{0}$ denote projection onto $T M_{0}$. Then

$$
\begin{aligned}
P_{0} A(v, 0, \ldots, 0)_{\left(x_{0}, x_{1}, \ldots, x_{k}\right)} & =P_{0} P_{\gamma} A(v, 0, \ldots, 0)_{\left(x_{00}, \ldots, x_{k}\right)} \\
& =P_{0} A P_{\gamma}(v, 0, \ldots, 0)_{\left(x_{0}, \ldots, x_{k}\right)} \\
& =P_{0} A(v, 0, \ldots, 0)_{\left(x_{0}, x_{1}^{\prime}, \ldots, x_{k}^{\prime}\right) .}
\end{aligned}
$$

Therefore we may define a parallel tensor $A_{0}$ of type $(1,1)$ on $M_{0}$ by $A_{0}\left(v_{x_{0}}\right)=$ $P_{0} A(v, 0, \ldots, 0)_{\left(x_{0}, x_{1}, \ldots, x_{k}\right)}$ where $x_{1}, \ldots, x_{k}$ are arbitrary points of $M_{1}, \ldots, M_{k}$ respectively. We similarly construct parallel tensors $A_{j}$ on $M_{j}, j=1,2, \ldots, k$ and it is clear that $A=A_{0} \times A_{1} \times \cdots \times A_{k}$.

REMARKS. (i) If $M$ is a complete simply connected Kaehler manifold then Proposition 4.5 implies that each of the irreducible factors of the de Pham decomposition of $M$ is Kaehler. Indeed, the proof of Proposition 4.5 is essentially the proof of the de Rham decomposition for Kaehler manifolds given in [11]. 
(ii) Proposition 4.5 may be used to prove the well-known result that a Riemannian manifold with parallel Ricci tensor is locally the product of Einstein manifolds.

Proposition 4.6. Let $A$ be a rank $r$ parallel tensor of type $(1,1)$ on a complete, simply connected Riemannian manifold $M$. If $A$ has no nonzero eigenvalues and if either (i) $A$ is normal or (ii) the de Rham decomposition has no Euclidean factor, then $M$ splits as the Riemannian product $M=N_{1} \times N_{2}$ with $N_{1}$ an $r$ dimensional Kaehler manifold and with $A$ nonsingular on $N_{1}$ and zero on $N_{2}$.

Proof. Let $M=M_{0} \times M_{1} \times \cdots \times M_{k}$ denote the de Rham decomposition of $M$ with $A=A_{0} \times A_{1} \times \cdots \times A_{k}$ the splitting given by Proposition 4.5. It follows from Proposition 4.1 that if $A_{j}$ is nonzero for some $j \geqslant 1$ then $M_{j}$ possesses a Kaehler structure and $A_{j}$ is nonsingular.

Now if $L$ is a normal linear transformation on a finite dimensional inner product space $V$, then it may be checked that the orthogonal complement of $\operatorname{Ker} L$ is $\operatorname{Im} L$. Thus on $M_{0}$, $\operatorname{Ker} A_{0}$ and $\operatorname{Im} A_{0}$ are two orthogonal parallel distributions with $T M_{0}=\operatorname{Ker} A_{0}+\operatorname{Im} A_{0}$. The dimension of $\operatorname{Im} A_{0}$ is even since the restriction of $A_{0}$ to $\operatorname{Im} A_{0}$ is nonsingular and $A_{0}$ has no nonzero eigenvalues. Fix a point $x_{0} \in M_{0}$ and let $H_{1}$ equal the leaf through $x_{0}$ of $\operatorname{Im} A_{0}$ and let $H_{2}$ equal the leaf through $x_{0}$ of $\operatorname{Ker} A_{0}$. Since $M_{0}$ is a Euclidean space it is clear that $M_{0}$ is isometric to $H_{1} \times H_{2}$ with $A_{0}$ zero on $H_{2}$ and nonsingular on $H_{1} . H_{1}$ is a Kaehler manifold since $H_{1}$ is an even dimensional Euclidean space. It is now clear how to construct the manifolds $N_{1}$ and $N_{2}$ in cases (i) and (ii).

In [6] the authors consider the concept of a framed metric $f$-manifold which among other things is a Riemannian manifold $M$ together with a skew-symmetric tensor $f$ of type $(1,1)$ satisfying $f^{3}+f=0$. A framed metric $f$-manifold is said to be covariant constant if a number of tensors including $f$ are parallel. As a corollary of Proposition 4.6 we strengthen slightly Theorem 14 of [6].

COROLlaRY 4.7. Let $M$ be a complete, simply connected, framed metric f-manifold such that $f$ is parallel. Then $M$ is isometric to a Riemannian product $N_{1} \times N_{2}$ where $N_{1}$ is a Kaehler manifold of dimension rank $f$ and $f=0$ on $N_{2}$.

\section{REFERENCES}

1. M. Berger, P. Gauduchon and E. Mazet, Le spectre d'une variété Riemannienne, Springer-Verlag, Berlin and New York, 1971.

2. I. Bivens, Codazzi tensors and reducible submanifolds, Global Differential Geometry and Global Analysis, Springer-Verlag, Berlin and New York, 1981.

3. B. Y. Chen and K. Yano, Integral formulas for submanifolds and their applications, J. Differential Geom. 5 (1971), 467-477.

4. R. Gardner, The technique of integral formulas in the geometry of immersions, Oberwolfach 4 (1971), 127-144.

5. $1-35$.

6. S. Goldberg and K. Yano, Globally framed f-manifolds, Illinois J. Math. 15 (1971), 456-474.

7. A. Gray, A note on manifolds whose holonomy group is a subgroup of $\mathrm{Sp}(n) \cdot \mathrm{Sp}(1)$, Michigan Math. J. 16 (1969), 125-128.

8. C. C. Hsiung, Some integral formulas for closed hypersurfaces, Math. Scand. 2 (1954), 286-294. 
9. S. Kobayashi, On compact Kaehler manifolds with positive Ricci tensor, Ann. of Math. 74 (1961), 570-574.

10. S. Kobayashi and K. Nomizu, On automorphisms of a Kaehlerian structure, Nagoya Math. J. 11 (1957), 115-124.

11. F_ Foundations of differential geometry, Interscience, New York, 1963.

12. J. Moore, Isometric immersions of Riemannian products, J. Differential Geom. 5 (1975), 159-168.

13. R. Reilly, On the first eigenvalue of the Laplacian for compact submanifolds of Euclidean space, Comment. Math. Helv. 52 (1977), 525-533.

Department of Mathematics, Rice University, Houston, Texas 77001 